\title{
Evaluation of CSF virological escape in patients on long-term PI monotherapy
}

Alejandro Arenas-Pinto, Wolfgang Stöhr, Amanda Clarke, lan Williams, Nicholas J Beeching, Jane Minton, Vincent Lee, Nicholas I Paton, the Protease Inhibitor monotherapy Versus Ongoing Triple therapy (PIVOT) CNS sub-study Team

Antiviral Therapy 2017; 10.3851/IMP3146

Submission date Acceptance date Publication date 17th November 2016

9th February 2017

24th February 2017

This provisional PDF matches the article and figures as they appeared upon acceptance.

Copyedited and fully formatted PDF and full text (HTML) versions will be made available soon.

For information about publishing your article in Antiviral Therapy go to http://www.intmedpress.com/index.cfm?pid=12 


\title{
Short communication
}

\section{Evaluation of CSF virological escape in patients on long-term PI monotherapy}

\author{
Alejandro Arenas-Pinto ${ }^{1,2 \star}$, Wolfgang Stöhr ${ }^{1}$, Amanda Clarke ${ }^{3}$, Ian Williams ${ }^{2}$, \\ Nicholas J Beeching ${ }^{4}$, Jane Minton ${ }^{5}$, Vincent Lee ${ }^{6}$, Nicholas I Paton ${ }^{1,7}$, the Protease \\ Inhibitor monotherapy Versus Ongoing Triple therapy (PIVOT) CNS sub-study \\ Team $^{+}$ \\ ${ }^{1}$ MRC-Clinical Trials Unit at UCL, London, UK \\ ${ }^{2}$ UCL Research Department of Infection and Population Health, London, UK \\ ${ }^{3}$ The Lawson Unit, Brighton and Sussex University Hospitals NHS Trust, Brighton, UK \\ ${ }^{4}$ Tropical \& Infectious Disease Unit, Royal Liverpool University Hospital, Liverpool, UK \\ 'Infection and Travel Medicine Services, St James' University Hospital, Leeds, UK \\ ${ }^{6}$ The Northern Sexual Health, Contraception \& HIV Service, Royal Infirmary, Manchester, UK \\ ${ }^{7}$ Yong Loo Lin School of Medicine, National University of Singapore, Singapore \\ *Corresponding author e-mail: a.arenas-pinto@ucl.ac.uk \\ †The members of the PIVOT CNS sub-study Team are listed in the Acknowledgements section
}

\section{Abstract}

Background: A strategy of protease inhibitor (PI) monotherapy with reintroduction of triple therapy in those who rebound has been shown to be a safe and effective treatment simplification approach for long-term management. We sought evidence for cerebrospinal fluid (CSF) virological escape in patients on long-term PI monotherapy.

Methods: We performed lumbar puncture in asymptomatic participants with suppressed plasma HIV-RNA after 96 weeks on the PI monotherapy arm (PImono) of the PIVOT trial. We also report CSF HIV-RNA concentration in trial participants who were investigated for neurological/neurocognitive symptoms during the trial regardless of study arm allocation.

Results: All 11 asymptomatic participants on PI-mono who were tested had undetectable CSF HIV-RNA at week 96. One of the 3 symptomatic participants on PI-mono had CSF HIV-RNA of 1895 copies/mL (undetectable in plasma), and neither of 2 symptomatic participants on triple therapy had CSF HIV-RNA detected.

Conclusions: CSF virological escape appears rare in asymptomatic patients on PI monotherapy and may not warrant routine CSF monitoring, but patients with symptoms merit more concern. 


\section{Introduction}

Evidence from a number of randomised controlled trials supports the safety and efficacy of using monotherapy with ritonavir boosted protease inhibitor (PI-mono) for treatment simplification in effectively suppressed HIV-positive patients with regular viral load monitoring and prompt reintroduction of combination therapy when needed [1-4]. However, concerns have been expressed about the possibility of occult viral replication in the central nervous system whilst it is suppressed in plasma (HIV-RNA $<50$ copies $/ \mathrm{mL}$ ) leading to cerebrospinal fluid (CSF) "virological escape". This is not an uncommon phenomenon even in patients without neurological symptoms on standard combination anti-retroviral therapy (cART) $[5,6]$ in whom it appears to be more frequent in patients with previous treatment interruption [5].

Here we report the findings of a prospective sub-study to evaluate CSF viral replication in patients taking part in the PIVOT trial, a large randomised controlled trial investigating the long-term outcomes of $\mathrm{PI}$ monotherapy treatment [1]. The aim of this sub-study was to estimate the proportion with detectable HIV-RNA in CSF amongst asymptomatic participants after 96 weeks on PI-mono. We also sought to gather data on symptomatic participants (regardless of their study group allocation) that required lumbar puncture (LP) for the investigation of neurological symptoms at any time during the study.

\section{Methods}

Participants were taking part in the PIVOT trial in which HIV positive adults on standard CART with no history of virological failure were randomised to either continuing ongoing triple therapy (OT, $N=291$ ) or to be switched to ritonavir-boosted PI monotherapy (PI-mono, $\mathrm{N}=296$ ) and followed up for a median of 44 months [1,7]. In this sub-study we collected data on CSF HIV-RNA in three groups: (i) neurologically asymptomatic participants randomised to and taking PI-mono who agreed to have a LP performed; (ii) participants taking PI-mono or (iii) standard CART with neurological or neurocognitive symptoms that led to LP being performed for their clinical investigation. Asymptomatic participants on $\mathrm{PI}$-mono from five of the study sites were invited to participate in this sub-study at their week 96 study visit. Symptomatic patients followed at any of the 43 PIVOT sites were considered eligible at any point during the study follow-up.

Magnetic resonance imaging scans (MRI) were performed in all participants before their LP. Detailed clinical information regarding neurological and neurocognitive complaints (i.e. headache, dizziness, visual disturbances, concentration or memory problems, and other cognitive complaints) was collected in all participants at the sub-study visit. The sub-study was approved by the Cambridge 4 Ethics Committee and all participants gave written informed consent. 


\section{Results}

Approximately 88 participants on PI-mono were approached at 5 sub-study sites for the crosssectional week 96 evaluation, of whom 11 consented to undergo LP (all were cognitivelyasymptomatic and on darunavir/ritonavir (DRV/r)) and were recruited; the MRI was normal in all cases and none had CSF HIV-RNA >50 copies/mL. In PIVOT, there were 30 grade $3 / 4$ CNS adverse events in 26 participants [1]. Of these, five symptomatic participants were recruited and had an LP performed during the trial (at weeks 87 - 109). MRI was normal in all cases. Of the three symptomatic participants on PI monotherapy (DRV/r), one had CSF HIV-RNA $>50$ copies/mL (described below). The other two symptomatic participants were on the CART arm of the trial (one on TRU/atazanavir (ATV)/r and one on abacavir/lamivudine/nevirapine) and neither had CSF HIV-RNA >50 copies/mL.

Our participant with CSF escape was male, infected with a wild-type virus (on sequencing), presented at age 49 years with an HIV seroconversion illness and was given 12 weeks of CART at the time. Four years later, at a CD4 count of 235 cell/ $/ \mathrm{mm}^{3}$ he was started on truvada (TRU)/DRV/r, following which his plasma HIV-RNA remained consistently $<50$ copies $/ \mathrm{mL}$. After 20 months he entered the PIVOT trial, was randomised to the PI-mono arm, and stopped TRU to continue on DRV/r alone. His HIV-RNA remained $<50$ copies $/ \mathrm{mL}$ on all tests during the trial (every 3-4 months). At week 96 he reported severe memory and concentration impairment and had an MRI scan (reported as normal) and an LP performed. His CSF HIV-RNA was 1895 copies $/ \mathrm{mL}$, no genotyping resistance test performed. At week 123 he reinstated triple therapy by adding TRU. Cognitive symptoms persisted and he underwent a second LP at week 135 (CSF HIV-RNA $<50$ copies $/ \mathrm{mL}$ ). Plasma HIV-RNA was $<50$ copies/mL throughout the entire follow-up including at the time of the initial detectable CSF HIVRNA. He was well at week 192 (end of the trial) but his cognitive complaints persisted.

\section{Discussion}

Although our sample size is relatively small (reflecting the fact that only a minority of asymptomatic participants were willing to join a research sub-study that involves an LP) our findings nevertheless make a significant contribution to the existing sparse literature on HIV-CSF escape amongst asymptomatic patients on PI monotherapy. Our finding that none of our asymptomatic participants had detectable HIV-RNA in the CSF, taken together with the data from other studies $[8,9]$ increases the confidence that HIV-CSF escape is a rare phenomenon in patients on PI monotherapy who are free from neurological and cognitive symptoms. All of our asymptomatic patients were taking DRV/r monotherapy, and we cannot therefore specifically comment on CSF viral escape in those on LPV/r or ATV/r monotherapy. Although we did not find any evidence of a difference in systemic VL suppression between the different PIs used as monotherapy [10], this does not necessarily hold for CSF viral escape as the CNS penetration of the drugs may differ [11]. In the MOST trial, there were 4 patients randomised to LPV/r monotherapy that were reported with CSF virological escape [12]. Kahlert et al recently published their experience on long-term efficacy of $\mathrm{PI}$ monotherapy in patients initially recruited into randomised controlled trials (RCTs). Four of 27 patients (15\%) had detectable HIV-RNA 
in CSF but undetectable viraemia [13], which seems to be consistent with overall findings from PI monotherapy randomised trials on LPV/r or ATV/r $[12,14,15]$. Of interest, no difference on virological escape was found between patients on PI monotherapy or standard combination ART in a crosssectional study ( $n=52)$ limited to cognitively impaired [16].

Our patient with neurocognitive symptoms who had evidence of CSF virological escape is similar to the two participants on DRV/r monotherapy in the MONOI trial who were investigated due to neurological symptoms (seizures and headache) [17] but not to the patient with virological failure (i.e. detectable HIV-RNA both in plasma and CSF) in the PROTEA trial who was asymptomatic [18]. In the MOST trial, 5 of the participants on LPV/r monotherapy were also neurologically symptomatic at the time of failing [12]. Taken together, the findings from the various studies including ours, suggest that in patients who have neuro-cognitive symptoms on PI monotherapy CSF viral escape is not uncommon. However, in most of those described in the previous studies, CSF HIV-RNA returned to undetectable levels after re-intensification with triple therapy $[12,17-19]$. In none of the cases of CSF viral escape while on PI monotherapy resistance been detected in the virus in the CSF (although not all were sequenced) and it is therefore unlikely that this would be a source for systemic resistance and viral rebound. Although most neurological symptoms tend to improve after ART optimisation in the case of CSF escape [19], the cognitive symptoms may persist in spite of re-suppression of viral load with re-introduction of triple therapy [8]. This may merely indicate that the symptoms are in part independent of the CSF HIV-RNA. Neurocognitive complaints are not uncommon in HIV positive patients, even if on effectively suppressive combination ART $[20,21]$ and tend to persist in the longterm [22].

Aside from CSF viral escape, patients on PI monotherapy may have CNS viral replication accompanying systemic HIV-RNA rebound as indeed may patients with systemic viral replication on triple therapy. Assessing this was not the purpose of this sub-study. In the main PIVOT trial we found a much higher rate of plasma HIV-RNA rebound in the PI-mono arm than the OT arm, but this was usually low-level, short-lived (re-suppressed rapidly with introduction of triple therapy) and there were no differences seen overall in CNS events or neurocognitive function between the two groups $[1,7]$. This suggests that if the systemic viral replication were accompanied by CSF viral replication this did not add significantly to the CNS morbidity overall.

The clinical management implications of this sub-study, taking into account the main trial findings and other PI monotherapy studies, are that asymptomatic patients on PI monotherapy may not need LP monitoring as CSF VL escape appears very rare. However, an LP should be considered in patients with persistent symptoms on PI monotherapy, although simple re-introduction of triple therapy as a precaution could be done and further investigation pursued only if the symptoms did not resolve. Longitudinal data are needed to better understand the mechanisms involved in CSF HIVRNA escape and the clinical implications of this phenomenon.

\section{Acknowledgements}

We thank all the patients and staff from all the centres participating in the PIVOT CNS sub-study. 
This analysis was funded by an unconditional scientific grant from Janssen Cilag Ltd. PIVOT was funded by the National Institute for Health Research Health Technology Assessment programme (project number 06/403/90). The views and opinions expressed herein are those of the authors and do not necessarily reflect those of the HTA programme, NIHR, NHS or the Department of Health.

\section{The PIVOT CNS sub-study Team are:}

Participating Sites: Elton John Centre, Brighton: M Fisher, A Clarke, W Hadley, D Stacey. Mortimer Market Centre, London: L Haddow, I Williams, N De Esteban, P Pellegrino, A Arenas-Pinto. Royal Liverpool University Hospital, Liverpool: N J Beeching, R Tamaklo. St James' Hospital, Leeds: J. J Minton, J Calderwood. Royal Infirmary, Manchester, UK: V Lee, K Patel, R Howe.

MRC Clinical Trials Unit at UCL: Nicholas Paton, Alejandro Arenas-Pinto, Wolfgang Stöhr, Karen Scott, David Dunn, Karen Sanders.

PIVOT Trial Steering Committee: Andrew Freedman (Chair), Ben Cromarty, Danielle Mercey, Sarah Fidler, Estee Torok, Abdel Babiker, Brian Gazzard, Chloe Orkin, Nicholas Paton.

PIVOT Data Monitoring Committee: Tim Peto (Chair), David Lalloo, Andrew Phillips and Robert James.

\section{Disclosure Statement:}

Authors declare no conflict of interest.

\section{References}

1. Paton NI, Stohr W, Arenas-Pinto A, et al. Protease inhibitor monotherapy for long-term management of HIV infection: a randomised, controlled, open-label, non-inferiority trial. Lancet HIV 2015; 2:e417-e426.

2. Mathis S, Khanlari B, Pulido F, et al. Effectiveness of protease inhibitor monotherapy versus combination antiretroviral maintenance therapy: a meta-analysis. PLoS One 2011; 6:e22003.

3. Pulido F, Arribas JR, Delgado R, et al. Lopinavir-ritonavir monotherapy versus lopinavirritonavir and two nucleosides for maintenance therapy of HIV. AIDS 2008; 22:F1-F9.

4. Arribas JR, Horban A, Gerstoft J, et al. The MONET trial: darunavir/ritonavir with or without nucleoside analogues, for patients with HIV RNA below 50 copies/ml. AIDS 2010; 24:223-230.

5. Eden A, Fuchs D, Hagberg L, et al. HIV-1 viral escape in cerebrospinal fluid of subjects on suppressive antiretroviral treatment. J Infect Dis 2010; 202:1819-1825.

6. Rawson T, Muir D, Mackie NE, Garvey LJ, Everitt A, Winston A. Factors associated with cerebrospinal fluid HIV RNA in HIV infected subjects undergoing lumbar puncture examination in a clinical setting. J Infect 2012; 65:239-245.

7. Paton NI, Stohr W, Oddershede L, et al. The Protease Inhibitor Monotherapy Versus Ongoing Triple Therapy (PIVOT) trial: a randomised controlled trial of a protease inhibitor monotherapy strategy for long-term management of human immunodeficiency virus infection. Health Technol Assess 2016; 20:1-158.

8. Ferretti F, Bigoloni A, Passeri L, et al. Cerebrospinal fluid analysis for HIV replication and biomarkers of immune activation and neurodegeneration in long-term atazanavir/ritonavir monotherapy treated patients. Medicine (Baltimore) 2016; 95:e4144.

9. Tiraboschi J, Hamzah L, Teague A, et al. The Impact of Switching from Atripla to Darunavir/Ritonavir Monotherapy on Neurocognition, Quality of Life, and Sleep: Results from a Randomized Controlled Study. AIDS Res Hum Retroviruses 2016.

10. Stohr W, Dunn DT, Arenas-Pinto A, et al. Factors associated with virological rebound in HIVinfected patients receiving protease inhibitor monotherapy. AIDS 2016; 30:2617-2624.

11. Delille CA, Pruett ST, Marconi VC, et al. Effect of protein binding on unbound atazanavir and darunavir cerebrospinal fluid concentrations. J Clin Pharmacol 2014; 54:1063-1071.

12. Gutmann C, Cusini A, Gunthard HF, et al. Randomized controlled study demonstrating failure of LPV/r monotherapy in HIV: the role of compartment and CD4-nadir. AIDS 2010; 24:2347-2354. 
13. Kahlert C, Bregenzer A, Gutmann C, et al. Late treatment failures in cerebrospinal fluid in patients on long-term maintenance ART with ritonavir-boosted protease PI monotherapy. Infection 2016; 44:329-335.

14. Vernazza P, Daneel S, Schiffer V, et al. The role of compartment penetration in PImonotherapy: the Atazanavir-Ritonavir Monomaintenance (ATARITMO) Trial. AIDS 2007; 21:1309_ 1315.

15. Santos JR, Llibre JM, Bravo I, et al. Short Communication: Efficacy and Safety of Treatment Simplification to Lopinavir/Ritonavir or Darunavir/Ritonavir Monotherapy: A Randomized Clinical Trial. AIDS Res Hum Retroviruses 2016; 32:452-455.

16. Estebanez M, Stella-Ascariz N, Mingorance J, et al. A comparative study of neurocognitively impaired patients receiving protease inhibitor monotherapy or triple-drug antiretroviral therapy. $J$ Acquir Immune Defic Syndr 2014; 67:419-423.

17. Katlama C, Valantin MA, Algarte-Genin M, et al. Efficacy of darunavir/ritonavir maintenance monotherapy in patients with HIV-1 viral suppression: a randomized open-label, noninferiority trial, MONOI-ANRS 136. AIDS 2010; 24:2365-2374.

18. Antinori A, Clarke A, Svedhem-Johansson V, et al. Week 48 efficacy and central nervous system analysis of darunavir/ritonavir monotherapy versus darunavir/ritonavir with two nucleoside analogues. AIDS 2015; 29:1811-1820.

19. Ferretti F, Gisslen M, Cinque P, Price RW. Cerebrospinal Fluid HIV Escape from Antiretroviral Therapy. Curr HIVIAIDS Rep 2015; 12:280-288.

20. Heaton RK, Clifford DB, Franklin DR, Jr., et al. HIV-associated neurocognitive disorders persist in the era of potent antiretroviral therapy: CHARTER Study. Neurology 2010; 75:2087-2096.

21. Simioni S, Cavassini M, Annoni JM, et al. Cognitive dysfunction in HIV patients despite longstanding suppression of viremia. AIDS 2010; 24:1243-1250.

22. Sacktor N, Skolasky RL, Seaberg E, et al. Prevalence of HIV-associated neurocognitive disorders in the Multicenter AIDS Cohort Study. Neurology 2016; 86:334-340. 